\title{
Investigating the cortical regions involved in MEP modulation in tDCS
}

\author{
Ricardo Salvador*, Cornelia Wenger and Pedro C. Miranda \\ Department of Physics, Faculdade de Ciências, Institute of Biophysics and Biomedical Engineering, Universidade de Lisboa, \\ Lisboa, Portugal
}

Transcranial magnetic stimulation (TMS) is used in several studies to evaluate cortical excitability changes induced by transcranial direct current stimulation (tDCS) of the primary motor cortex. Interpretation of these results, however, is hindered by the very different spatial distribution of the electric field (E-field) induced by the two techniques and by the different target neurons that they might act upon. In this study we used the finite element method to calculate the E-field distribution induced by TMS and TDCS in a realistically shaped model of a human head. A model of a commercially available figure-8 coil was placed over a position above the identified hand knob $(H K)$ region. We also modeled two configurations of bipolar tDCS montages with one of the electrodes placed over the HK and a return electrode over the contralateral orbital region. The electrodes over the HK were either rectangular in shape, with an area of $35 \mathrm{~cm}^{2}$ or cylindrical with an area of $\pi \mathrm{cm}^{2}$ (1 $\mathrm{cm}$ radius). To compare the E-field distribution in TMS and the two tDCS models, average values of the E-field's magnitude as well as the polar and azimuthal angle were investigated in the HK region and premotor areas. The results show that both techniques induce fields with different magnitudes and directions in the

OPEN ACCESS

Edited by:

Michael A. Nitsche,

Georg-August-University, Germany

Reviewed by:

Axel Thielscher,

Copenhagen University Hospital

Hvidovre, Denmark

Marom Bikson,

The City College of New York, USA

*Correspondence:

Ricardo Salvador

rnsalvador@ciencias.ulisboa.pt

Received: 26 June 2015

Accepted: 24 September 2015

Published: 13 October 2015

Citation:

Salvador $R$, Wenger $C$ and Miranda PC (2015) Investigating the cortical regions involved in MEP modulation in $T D C S$.

Front. Cell. Neurosci. 9:405. doi: 10.3389/fncel.2015.00405
HK: the field in tDCS is predominantly perpendicular to the cortical surface, contrary to what happens in TMS where the field is mostly parallel to it. In the premotor areas, the magnitude of the E-field induced in TMS was well below the accepted threshold for MEP generation, $100 \mathrm{~V} / \mathrm{m}$. In tDCS, the magnitude of the field in these areas was comparable to that induced at the HK with a significant component perpendicular to the cortical surface. These results indicate that tDCS and TMS target preferentially different neuronal structures at the HK. Besides, they show that premotor areas may play a role in the tDCS-induced after effects on motor cortex excitability.

Keywords: transcranial magnetic stimulation, TMS, transcranial direct current stimulation, tDCS, motor cortex, finite element modeling, motor evoked potential

\section{INTRODUCTION}

Transcranial magnetic stimulation (TMS) is used in a number of studies to evaluate cortical excitability changes induced by transcranial direct current stimulation (tDCS; Nitsche et al., 2008). One cortical area studied extensively is the primary motor cortex (M1; Nitsche and Paulus, 2000). In these studies an initial assessment of the optimal position for stimulation of M1 is performed with TMS and then the average value of the magnitude of the motor evoked potential (MEP) in a target muscle is determined. After this stage, tDCS is applied at the same position for a given period of time, after which the average MEP size following TMS is again measured to determine 
changes relative to baseline. The results show that anodal tDCS of the M1 area results in an increased MEP size and that cathodal stimulation has the opposite effect (Nitsche and Paulus, 2000, 2001). The interpretation of these results, however, is not clear since the properties of the E-field induced by both techniques and, therefore, the mechanisms through each they act upon neurons are very different. The long-lasting (10-20 $\mathrm{min})$ and low intensity (1-2 mA) currents injected through the electrodes during tDCS bring about subthreshold membrane polarizations of the cellular body of pyramidal neurons in the cortex which cause synaptic plasticity changes (Liebetanz et al., 2002; Nitsche et al., 2003; Radman et al., 2009). The E-field induced during TMS has very distinct properties, since it has a much shorter duration (hundreds of microseconds) and a much higher magnitude ( $100 \mathrm{~V} / \mathrm{m}$; Roth et al., 1991; Kammer et al., 2001). It is thought to induce suprathreshold polarizations at bends and terminations of neurons in the cortex, provided they are correctly aligned with the applied E-field (Amassian et al., 1992; Nagarajan et al., 1993; Roth, 1994; Salvador et al., 2011).

A proper analysis of the results of this protocol requires, therefore, an accurate knowledge about the distribution of the E-field induced in the brain during both techniques. Since no direct in vivo measurements of the E-field in humans are possible, numerical techniques continue to be the only available means to predict the E-field distribution. Over the last few years several studies have been published describing numerical calculations of E-field distribution in realistic head models in both tDCS (Datta et al., 2009; Metwally et al., 2012; Miranda et al., 2013; Opitz et al., 2015) and TMS (Chen and Mogul, 2009; Opitz et al., 2011, 2013; Thielscher et al., 2011). However, to the best of our knowledge, no study has been conducted in which the field distribution in both techniques has been compared in the same head model. In this study we used the finite element (FE) method to numerically calculate the E-field induced in a realistic head model, built from magnetic resonance (MR) images of a single subject, during TMS and tDCS. In the analysis of the E-field in the cortex, we focused not only on the E-field's magnitude, but also on its direction. The results help shed light on the cortical areas targeted preferentially by both forms of stimulation.

\section{MATERIALS AND METHODS}

\section{Head Model}

A realistic head model was created from $\mathrm{T}_{1}$ - and $\mathrm{T}_{2}$-weighted images $\left(1 \mathrm{~mm}^{3}\right.$ isotropic resolution) of a 20 years old healthy female subject who agreed to participate in this study. The study was approved by the local ethical committee of Hospital Lusíadas, Lisbon, Portugal, where the images were obtained. Diffusion weighted images (DWI) were also acquired for the same subject $(1.25 \times 1.25 \times 3.5 \mathrm{~mm}$ resolution). The images were registered to MNI space and surface meshes of the different tissues were obtained from the anatomical images using an adapted version of the SimNibs pipeline (http://www.simnibs.de), and the software package Brainsuite (http://brainsuite.org). These surface meshes were then corrected with the software Mimics (v16.0, www.materialise.com), which was also used to generate tetrahedral volume meshes suitable for FE analysis. The resulting meshes were free of irregularities such as holes in the CSF, and regions where the skull touched the GM outer surface. The meshes have more than 3.5 million tetrahedral second order elements. The quality of the mesh in Comsol is quantified by the parameter element quality which varies between 0 (degenerated low quality element) and 1 (best possible element; Comsol, 2013a). The meshes used in this study had an average element quality $>0.4$. The surface meshes for the different tissues are shown in Figure 1A.

\section{Geometry and Placement of the Electrodes and Coils}

The head model was prepared for tDCS E-field calculations by adding two electrodes on the scalp (see Figures 1E,F). Two different bipolar electrode configurations were considered, both with the anode-a rectangular $5 \times 7 \mathrm{~cm}^{2}$ electrode or a circular one with a radius of $1 \mathrm{~cm}\left(\pi \mathrm{cm}^{2}\right)$-centered over the left handknob. The latter was identified based on anatomical landmarks, as described by Yousry et al. (1997). The position of the hand-knob in the model is shown in Figures 1B,C. The rectangular electrode was tilted $45^{\circ}$ from the anterior-posterior axis, which made its longer side approximately parallel to the central sulcus. The cathode in both cases was a $5 \times 7 \mathrm{~cm}^{2}$ electrode placed over the contralateral eyebrow. The inner surface of all electrodes followed perfectly the curvature of the scalp, modeling a perfect electrodescalp contact. All electrodes were modeled as homogeneous conducting media with a conductivity of $2 \mathrm{~S} / \mathrm{m}$, as was done in previous studies (Miranda et al., 2013). For the TMS calculations, the coil was also centered over the left hand-knob (Figure 1D). The coil replicates Magstim's $70 \mathrm{~mm}$ figure- 8 coil, which has nine windings and a $90 \mathrm{~mm}$ separation between wing centers (Thielscher and Kammer, 2002). The coil's wires were modeled as circular lines placed at a distance of $9 \mathrm{~mm}$ from the scalp. In accordance with usual practice, the plane of the coil was tangential to the scalp surface and its handle was rotated by $45^{\circ}$ from the anterior-posterior axis (Kammer et al., 2001).

\section{E-field Calculation}

All E-field calculations were performed using Comsol (v4.3b, www.comsol.com). To calculate the E-field distribution in tDCS, Laplace's equation subject to appropriate boundary conditions was solved to obtain the distribution of the scalar electric potential $(\phi)$ in the head model:

$$
\vec{\nabla} \cdot(\sigma \vec{\nabla} \phi)=0
$$

where $\sigma$ is the electrical conductivity tensor. The E-field was then obtained taking the gradient of the scalar potential:

$$
\vec{E}=-\vec{\nabla} \phi
$$

By default Comsol enforces the continuity of the normal component of the current density at the inner boundaries of the model, and an electrical insulation boundary condition at the outer surfaces. We additionally imposed a floating potential 




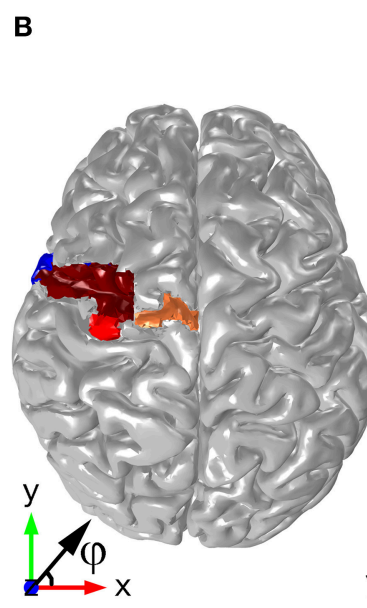

E

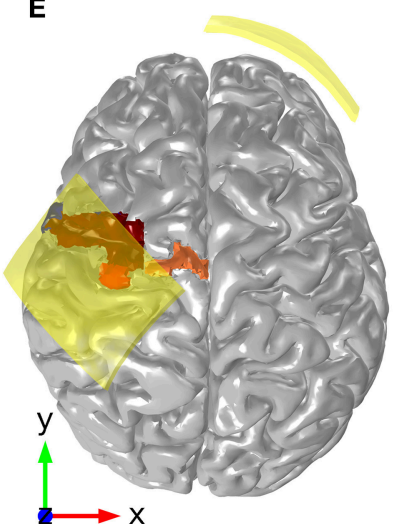

C

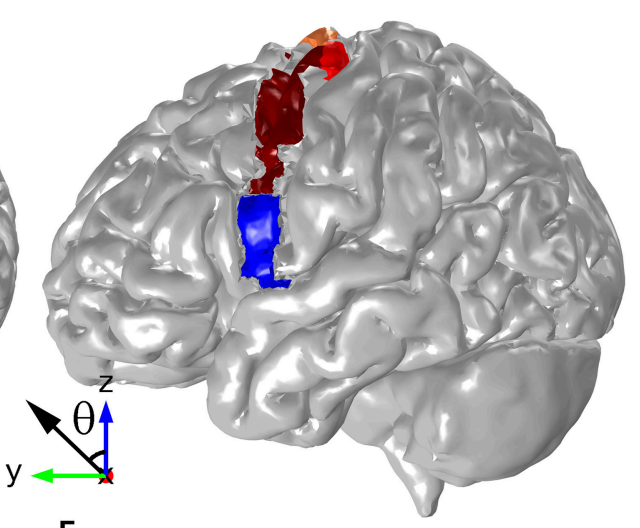

F

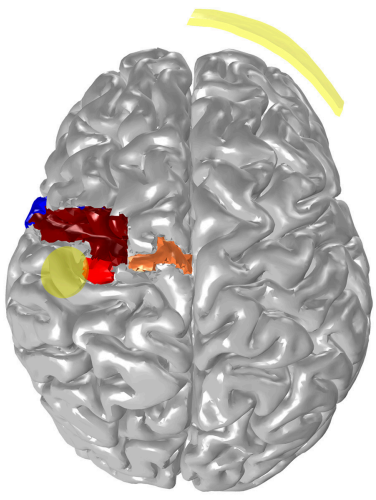

FIGURE 1 | Head model geometry, depicting the surfaces of the scalp, skull, CSF, GM, and WM (A). The position of motor and pre-motor areas are shown in panels (B,C): HK (red), SMA (orange), PMd (brown), and PMv (blue). The positions and orientations of the TMS coil, the electrodes in the montage with two $35 \mathrm{~cm}{ }^{2}$ electrodes and the ones in the montage with the $\pi \mathrm{cm}^{2}$ anode are shown in panels (D-F), respectively. The angles shown in panels (B,C) represent the azimuthal angle $(\varphi)$ and the polar angle $(\theta)$, respectively.

boundary condition at the outer surface of each electrode (excluding the lateral surfaces). This boundary condition automatically sets uniform voltages at the outer surfaces of both electrodes so that the surface integral of the component of the current density perpendicular to electrode's surface is equal to a user specified value: $1 \mathrm{~mA}$ for the anode and $-1 \mathrm{~mA}$ for the cathode.

The calculation of the field induced in TMS was slightly more complex since the E-field induced in this technique is actually the sum of two components: a primary component which only depends on coil geometry and a secondary component which depends also on the geometry of the head and its dielectric properties. These E-field components can be calculated from a magnetic vector potential $(\vec{A})$ and the scalar electric potential $(\phi):$

$$
\vec{E}=\vec{E}_{P r i}+\vec{E}_{S e c}=-\frac{d \vec{A}}{d t}-\vec{\nabla} \phi
$$

Calculation of the primary component was based on the analytical solution for the E-field induced by circular coils with idealized "line" wires, as described in a previous study (Tofts, 1990):

$$
A_{\varphi}=\frac{\mu_{0} I}{\pi k}\left(\frac{a}{\rho}\right)^{1 / 2}\left[K\left(k^{2}\right)\left(1-\frac{1}{2} \times k^{2}\right)-E\left(k^{2}\right)\right]
$$

where $A_{\varphi}$ is the azimuthal component of the magnetic potential, $\mu_{0}=4 \pi \times 10^{-7} \mathrm{H} / \mathrm{m}$ is the magnetic permeability, $I$ is the current in the coil, $a$ is the coil's radius, $\rho$ is the distance between the point where $A_{\varphi}$ is being calculated and the coil's axis, $k^{2}=$ $4 a \rho\left[(a+\rho)^{2}+z^{2}\right]^{-1}$ and $K$ and $E$ are elliptic integrals of the first and second kind, respectively. The primary E-field induced by the figure- 8 coil modeled in this work was then obtained by summing the individual contribution of each circular coil composing it. These analytical solutions were implemented in Matlab (v2013b, www.mathworks.com). This information was then imported into Comsol as a source term in Laplace's equation, which was used to calculate the secondary component of the E-field:

$$
\vec{\nabla} \cdot\left(\left(\sigma+j w \varepsilon_{0} \varepsilon_{r}\right) \vec{\nabla} \phi+\sigma \frac{d \vec{A}}{d t}\right)=0
$$


where $\varepsilon_{0}=8.8542 \times 10^{-12} \mathrm{~F} / \mathrm{m}$ is the vacuum permittivity, $\varepsilon_{r}$ is the relative permittivity and $w$, the angular frequency is equal to $2 \pi f$ where $f$ is the frequency of the current in the coil. The same boundary conditions that were discussed earlier for the tDCS calculation were also imposed to the inner and outer boundaries of this model. We assumed that the current in the coil varied sinusoidally with a frequency of $5 \mathrm{kHz}$ and a peak amplitude of $2133 \mathrm{~A}$, which yields a maximum value of $\mathrm{d} I / \mathrm{dt}$ of $67 \mathrm{~A} / \mu \mathrm{s}$. This corresponds to the average resting motor threshold (RMT) for this coil (Kammer et al., 2001).

The isotropic conductivity values of the tissues for both models were based on the ones used in previous studies (Miranda et al., 2013): $0.33 \mathrm{~S} / \mathrm{m}$ for the scalp and gray matter (GM), $0.008 \mathrm{~S} / \mathrm{m}$ for the skull, $1.79 \mathrm{~S} / \mathrm{m}$ for the cerebrospinal fluid (CSF), and $0.15 \mathrm{~S} / \mathrm{m}$ for the white matter (WM). Efield calculations were also performed taking into account the anisotropic conductivity of the WM and GM, as determined from DWI data. The latter were processed with the volumenormalized mapping approach of the SimNibs pipeline (Opitz et al., 2011) to find the conductivity values from the diffusion tensor components. In agreement with other studies (Thielscher et al., 2011), the relative dielectric permittivity of all tissues was set to one in the TMS model, which is equivalent to ignoring any capacitive effects of tissues in the calculation of the E-field (Roth et al., 1991). This approximation is considered valid for frequencies typical of TMS pulses (Plonsey and Heppner, 1967).

For the tDCS calculation, an iterative solver was used (BiCGStab) with the preconditioner Geometric Multigrid. The relative tolerance parameter, that determines when the solution has converged, was set at $10^{-3}$. For the TMS model, we used a different iterative solver (GMRES) with the preconditioner Incomplete $\mathrm{LU}$ (set with a drop-tolerance of $5 \times 10^{-4}$ ). The same relative tolerance of $10^{-3}$ was used in this calculation. For more details on these solvers the interested reader may refer to Comsol (2013b).

\section{RESULTS}

In order to analyze and compare the E-field distribution induced in the different configurations modeled in this study, we calculated average volume values for: the E-field's magnitude, the azimuthal angle (the angle between the projection of the E-field onto the axial plane and the $\mathrm{x}$-axis, i.e., the axis pointing from the left to the right hemisphere, Figure 1B) and the polar angle (the angle of the E-field with respect to the $\mathrm{z}$-axis, i.e., the inferiorsuperior axis, Figure 1C). These average values were calculated in the GM in regions identified as important in stimulation of the hand motor area (see Figures 1B,C): hand-knob (HK), whole motor cortex (M1), supplementary motor area (SMA) and lateral premotor cortex (ventral, PMv, and dorsal, PMd). Identification of these landmarks was based on descriptions available in the literature about their shape, position and boundaries (Dum and Strick, 1991; Mayka et al., 2006). In summary, we considered that the lateral premotor cortex (PMv and PMd) ranged between the anterior part of the precentral gyrus and the precentral sulcus (posterior-anterior direction), and the lateral sulcus and the virtual continuation of the superior frontal sulcus (lateral-medial direction). The boundary between the PMv and PMd was considered to be the virtual continuation of the inferior frontal sulcus. The SMA ranged between the medial boundary of the PMd and the central fissure (lateral-medial direction) and its ventral boundary was defined as the cingulate sulcus. Due to difficulties in precisely selecting all these regions in the model, and the lack of knowledge about their precise anatomical boundaries, there is some error associated with their definition. However, some of these errors are minimized by presenting average values for the figures calculated in this study. The latter are expected to be less sensitive to misrepresentations of the regions of interest considered here (the interested reader may refer to supplementary material for more data supporting this).

\section{Electric Field Distribution in TMS}

The main feature of the distribution of the magnitude of the Efield was the presence of two maxima on the cortical surface: an absolute maximum in the post-central gyrus and a local maximum in the pre-central gyrus (see Figures $2 \mathbf{A}, \mathbf{B}$ ). In the anisotropic model, the E-field on the post- and pre-central gyri reached 177 and $144 \mathrm{~V} / \mathrm{m}$, respectively. The field's magnitude was strongest under the coil windings and its value rapidly decreased with distance from the coil. In the central sulcus near the HK, the field was stronger in the posterior than in the anterior wall, and it decreased rapidly with depth (see Figure 2B).

The E-field induced in TMS was very localized in the regions located under the coil's center (see Figure 2A). As such the Efield's magnitude average value was stronger in the $\mathrm{HK}$ than in any pre-motor area, as can be seen in Table 1. In the HK, the average azimuthal angle was $44^{\circ}$ very similar to the orientation of the central section of the coil. Also in that region, the average polar angle was $77^{\circ}$. This suggests that the E-field induced in TMS is predominantly tangential to the cortical surface at the top of the pre-central gyrus, with only a small upward component, as is clearly shown in Figure 2B.

Changing the position of the coil $1 \mathrm{~cm}$ anteriorly (along the line between the two electrodes) led to only small changes in the field distribution. The overall maximum was still located at the top of the post-central gyrus and its value decreased only slightly to $175 \mathrm{~V} / \mathrm{m}$, as compared to $179 \mathrm{~V} / \mathrm{m}$ in the original model. The local maximum and the mean values over the HK also increased somewhat in the model with the translated coil: 10.6 and $8.3 \%$, respectively (values are expressed as a percentage of the value in the original model). This slight increase was also observed in all the pre-motor regions.

\section{Electric Field Distribution in tDCS}

In the tDCS model with the larger anode, the highest E-field values were found at the crowns of some of the gyri located closer to the electrodes' edges (see Figure $2 \mathrm{C}$ ). At the bottom of the sulci under the electrodes there were also small regions with very high E-field magnitude. In the cortical regions between the two electrodes and away from their edges, the local maxima were located at the top of the gyri. However, the E-field's magnitude in these regions was smaller than in the former regions. Contrary to what happened in the TMS model, the E-field induced during tDCS ( $35 \mathrm{~cm}^{2}$ anode) was stronger on the crown of the 

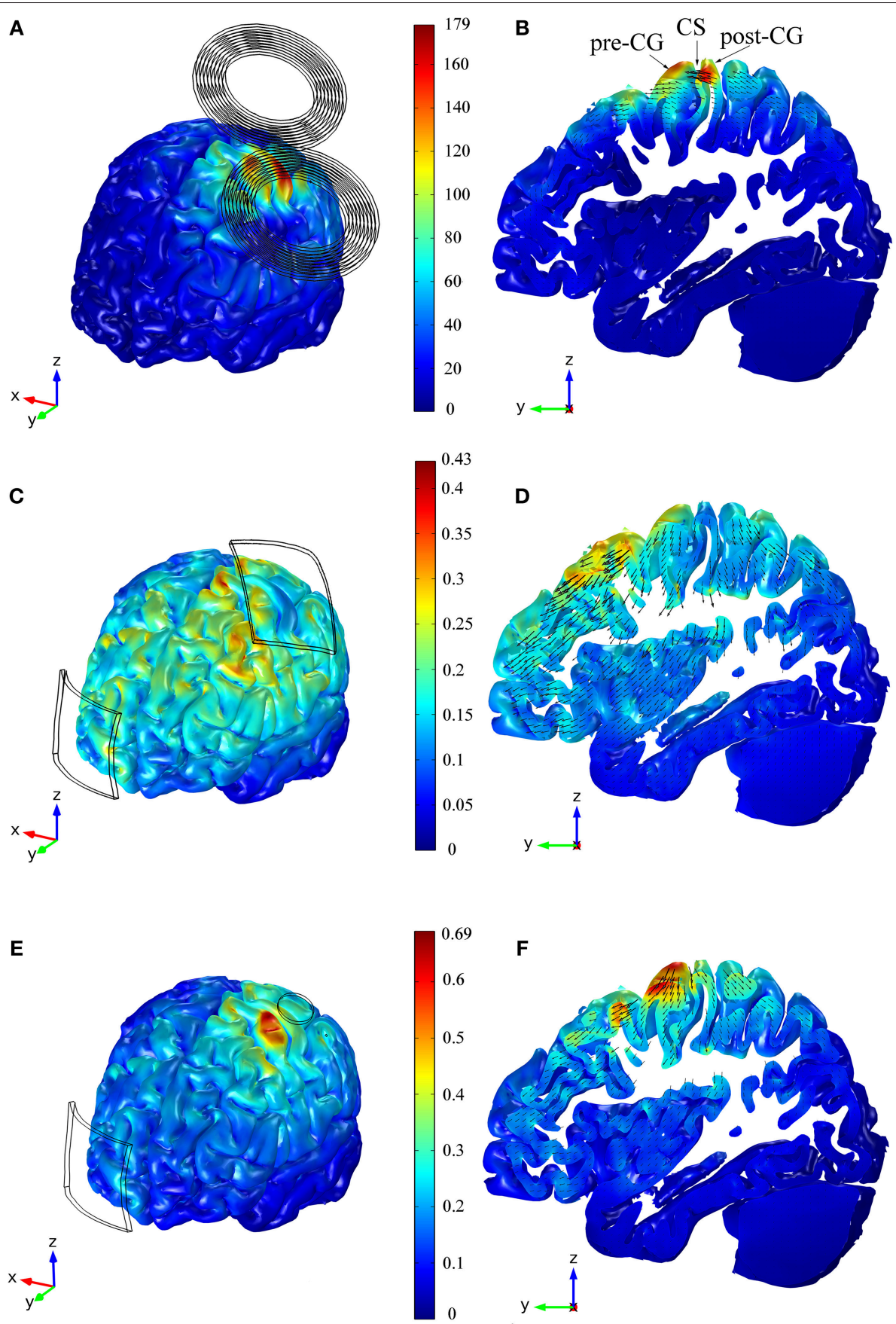

FIGURE 2 | Distribution of the E-field's magnitude induced during TMS (A,B), tDCS with the $35 \mathrm{~cm}^{2}$ electrode (C,D) and tDCS with the $\pi \mathrm{cm}^{2}$ anode $\mathbf{( E , F ) . ~ T h e ~ f i g u r e s ~ i n ~ t h e ~ l e f t ~ c o l u m n ~ s h o w ~ t h e ~ E - f i e l d ' s ~ m a g n i t u d e ~ i n ~ t h e ~ G M ~ v o l u m e , ~ w h e r e a s ~ t h e ~ f i g u r e s ~ i n ~ t h e ~ r i g h t ~ c o l u m n ~ s h o w ~ t h e ~ E - f i e l d ' s ~ d i s t r i b u t i o n ~ i n ~ a ~ s a g i t t a l ~}$ view of a slab of GM tissue with a thickness of $1.3 \mathrm{~cm}$ and passing through the $\mathrm{HK}$ and PMd regions. The figures within the same row share the same color scale (E-field's magnitude in $\mathrm{V} / \mathrm{m}$ ). The figures in the right column also show vector plots of the projection of the E-field in the depicted sagittal plane. Panel (B) also shows the location of the central sulcus (CS), pre and post-central gyrus (pre-CG/post-CG).

pre-central gyrus $(0.30 \mathrm{~V} / \mathrm{m})$ than on the crown of the postcentral gyrus $(0.10 \mathrm{~V} / \mathrm{m})$, as shown in Figure 2D. The global maximum of $0.43 \mathrm{~V} / \mathrm{m}$ occurred in very localized regions located on the gyri near the edges of the electrodes and at the bottom of the sulci beneath it. The field was again low on the walls of the central sulcus but had a local maximum at the bottom of the central sulcus that is comparable to the value at the crown of the pre-central gyrus.

In the model with the smaller anode, the global maximum was located on the crown of the pre-central gyrus, as shown in 
TABLE 1 | Average and maximum values of the E-field's magnitude, polar angle, azimuthal angle and angle between the field induced in tDCS and TMS $\left({ }^{T}{ }_{T M S}{ }^{\wedge} D C S\right)$ in the different regions of interest (ROIs).

\begin{tabular}{|c|c|c|c|c|c|c|c|c|c|c|c|c|c|c|}
\hline \multirow{3}{*}{$\begin{array}{l}\text { ROI } \\
\\
\text { HK }\end{array}$} & \multicolumn{6}{|c|}{ E-field's magnitude average|maximum (V/m) } & \multicolumn{3}{|c|}{ Polar angle $\left({ }^{\circ}\right)$} & \multicolumn{3}{|c|}{ Azimuthal angle $\left({ }^{\circ}\right)$} & \multicolumn{2}{|c|}{${ }^{\theta}{ }_{T M S}{ }^{\prime}{ }_{D C S}\left({ }^{\circ}\right)$} \\
\hline & \multicolumn{2}{|c|}{ TMS } & \multicolumn{2}{|c|}{$35 \mathrm{~cm}^{2}$} & \multicolumn{2}{|c|}{$\pi \mathrm{cm}^{2}$} & \multirow{2}{*}{$\begin{array}{c}\text { TMS } \\
76\end{array}$} & \multirow{2}{*}{$\begin{array}{l}35 \mathrm{~cm}^{2} \\
129\end{array}$} & \multirow{2}{*}{$\begin{array}{l}\pi \mathbf{c m}^{2} \\
136\end{array}$} & \multirow{2}{*}{$\begin{array}{c}\text { TMS } \\
44\end{array}$} & \multirow{2}{*}{$\begin{array}{l}35 \mathrm{~cm}^{2} \\
13\end{array}$} & \multirow{2}{*}{$\frac{\pi \mathbf{c m}^{2}}{17}$} & \multirow{2}{*}{$\frac{35 \mathrm{~cm}^{2}}{62}$} & \multirow{2}{*}{$\frac{\pi \mathbf{c m}^{2}}{68}$} \\
\hline & 65 & 144 & 0.16 & 0.29 & 0.34 & 0.74 & & & & & & & & \\
\hline SMA & 33 & 93 & 0.17 & 0.39 & 0.22 & 0.53 & 87 & 120 & 117 & 33 & 24 & 33 & 36 & 33 \\
\hline PMd & 41 & 146 & 0.19 & 0.36 & 0.22 & 0.69 & 85 & 128 & 129 & 53 & 39 & 53 & 52 & 53 \\
\hline PMv & 16 & 47 & 0.15 & 0.25 & 0.15 & 0.26 & 92 & 139 & 138 & 107 & 52 & 126 & 123 & 126 \\
\hline C & 7 & 19 & 0.15 & 0.37 & 0.15 & 0.38 & 149 & 100 & 100 & 74 & 52 & 53 & 91 & 91 \\
\hline
\end{tabular}


represents a circular regions located under the position of the cathode $\left(A=25 \mathrm{~cm}^{2}\right)$.

Figure 2E. This again corresponds to the position of the edge of the electrode. There, the maximum of the E-field's magnitude was higher than the global maximum obtained in the model with the larger anode: 0.69 and $0.43 \mathrm{~V} / \mathrm{m}$, respectively. At the bottom of the central sulci under the electrode there was again a local maximum of the E-field's magnitude: $0.40 \mathrm{~V} / \mathrm{m}$ (Figure 2F). At the top of the gyri between the two electrodes, away from the electrode's edges, the distribution of the E-field was similar in the two models. The E-field distribution under the cathode was also similar in both models, and this is reflected in similar average values in the row labeled $\mathrm{C}$ in Table $\mathbf{1}$.

The E-field induced during tDCS was less focal than the one induced during TMS, regardless of anode size. As a consequence, the average E-field's magnitude in pre-motor areas was comparable to the average E-field's magnitude in the $\mathrm{HK}$, particularly for the $35 \mathrm{~cm}^{2}$ electrode (see Table 1). The orientation of the E-field in these two regions, however, was very different. The field was essentially perpendicular to the cortical surface on the crown of the gyri under the anode and cathode, whereas it was predominantly tangential to the gyri between electrodes (see Figures 2D,F). In the PMd, since this region was still mostly covered by the large $35 \mathrm{~cm}^{2}$ placed over the HK, this resulted in average polar angles comparable to those obtained in the HK (see Table 1). This indicates that the field has a strong component perpendicular to the surface of the gyri. In the model with the $\pi \mathrm{cm}^{2}$ anode, the mean polar angle at the PMd was slightly smaller than that over the HK. At the $\mathrm{PMv}$, the opposite happened, with a higher mean polar angle than at the HK region. At the SMA, the mean polar angles were again lower than those over the HK which corresponds to an E-field with a weaker component perpendicular to the gyri in this region. The average azimuthal angle was low in the HK, indicating a strong left-right component. In the pre-motor areas, it increased to values closer to the azimuthal angle of the line defined by the two electrodes $\left(24^{\circ}-53^{\circ}\right.$, as shown in Table 1$)$. The exception occurred at the PMv in the model with the $\pi \mathrm{cm}^{2}$ anode, where the mean azimuthal angle was $126^{\circ}$ indicating a stronger right-left component.

For the model with the $\pi \mathrm{cm}^{2}$ anode the average field in the $\mathrm{HK}$ increased by $112.5 \%$ relative to the model with the $35 \mathrm{~cm}^{2}$ electrode. The average field in the premotor areas did not change much with electrode size: in the model with the smaller electrode, the mean E-field increased $29.4 \%$ at the SMA and $15.8 \%$ at the
PMd. At the PMv the average field was independent of electrode size. Regarding the variation of the average polar and azimuthal angles, they showed distinct behaviors: the average polar angle varied only slightly between the two models (maximum variation of $7^{\circ}$ at the $\mathrm{HK}$ ), whereas the average azimuthal angle increased significantly in all areas for the model with the smaller electrode (maximum variation of $74^{\circ}$ at the $\mathrm{PMv}$ ).

\section{Comparison between the Field Induced in tDCS and TMS}

In TMS an E-field magnitude of $100 \mathrm{~V} / \mathrm{m}$ is a well-established value for the threshold for MEP generation, in good agreement with our previous work (Salvador et al., 2011). The only regions where the E-field magnitude was greater than this value were the crown and lip of the pre- and post-central gyri. Increasing the stimulator output made the E-field magnitude reach values above threshold in more gyri. The same thing happened for a greater portion of the posterior wall of the central sulcus (see Figures $3 \mathbf{A}-\mathbf{C}$ ). The field in the anterior wall of the central sulcus, however, only reached threshold values very close to maximum stimulator output for the Magstim 200 stimulator (data not shown).

In tDCS there is no threshold value described in the literature for the onset of the excitability changes induced by the E-field. Therefore, we considered several possible threshold values from $0.1 \mathrm{~V} / \mathrm{m}$ to $0.3 \mathrm{~V} / \mathrm{m}$ (Figures $3 \mathrm{D}-\mathrm{I}$ ). A threshold level of $0.1 \mathrm{~V} / \mathrm{m}$ was attained in most regions under the electrodes and in between them, regardless of anode size (Figures 3D,G). Increasing the threshold value to $0.2 \mathrm{~V} / \mathrm{m}$ decreased the regions above threshold substantially (see Figures 3E,H), especially in the model with the $35 \mathrm{~cm}^{2}$ anode (Figure $3 \mathrm{E}$ ). In that model, the only regions above $0.2 \mathrm{~V} / \mathrm{m}$ were the crown of the pre-central gyrus and gyri located anterior to it. The bottom of the central sulcus was also above threshold. In the model with the smaller anode the regions above this threshold extended further, now including also the posterior wall of the central sulcus and gyri located posterior to the central sulcus (including the post-central gyrus). Regions above a threshold value of $0.3 \mathrm{~V} / \mathrm{m}$ were very localized in the model with the $35 \mathrm{~cm}^{2}$ anode (Figure 3F). In the model with the $\pi \mathrm{cm}^{2}$ anode, however, the regions above this threshold still occupied a great part of the pre-central gyrus and the posterior wall of the central sulcus, as well as the post-central gyrus and parts of gyri located anterior to the HK (Figure 3I). 


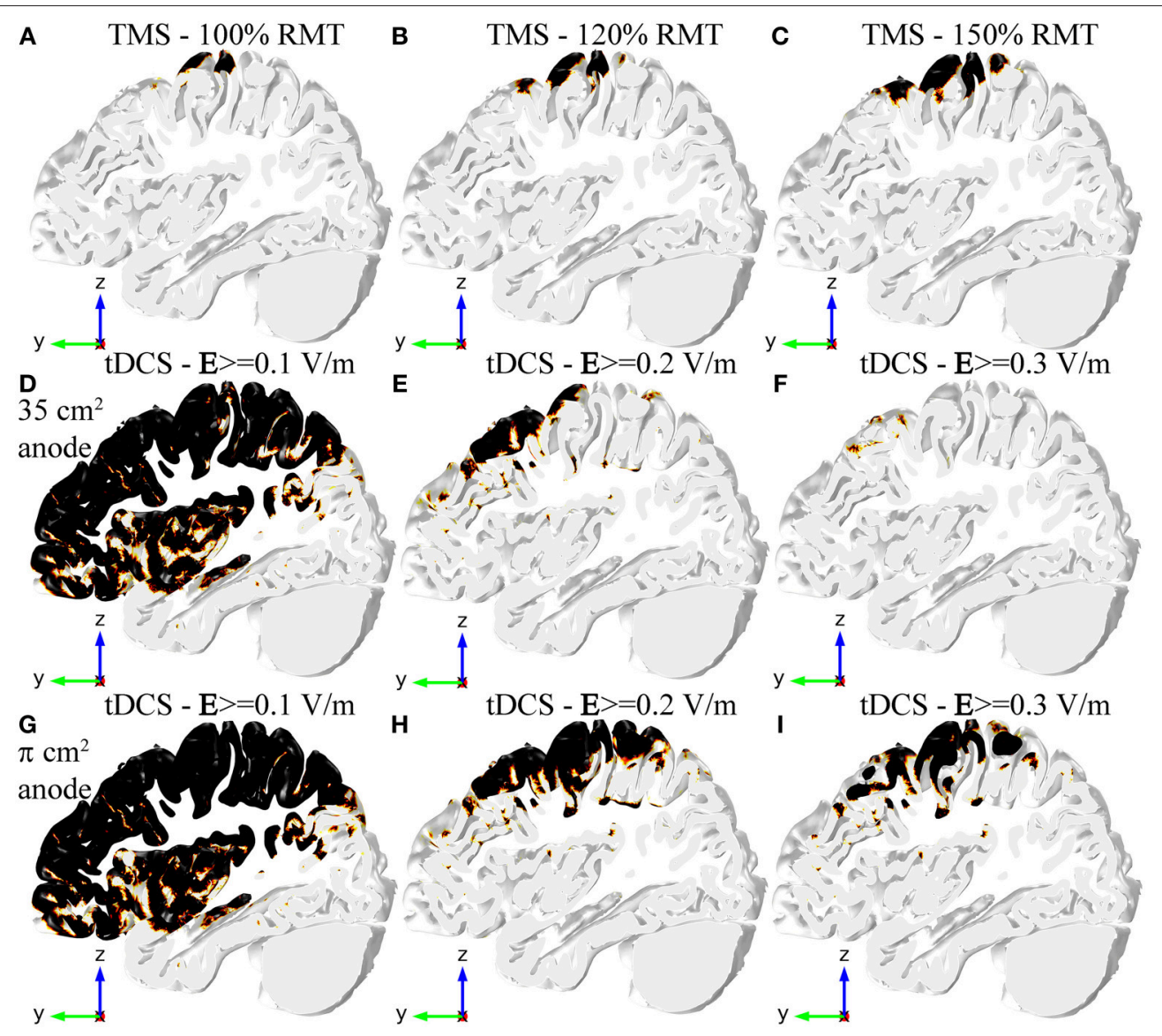

FIGURE 3 | Regions in the GM volume where the field is above a specified threshold in TMS (A-C), tDCS with the $35 \mathrm{~cm}^{2}$ electrode (D-F), and tDCS with the $\pi \mathbf{~ c m}^{2}$ anode (G-I). Regions shown in black are above threshold. The region selected is the same slab of GM tissue passing through the HK and PMd shown in Figure 2. The threshold for TMS was set to $100 \mathrm{~V} / \mathrm{m}$ in all figures, but the stimulator output was set to $100 \%$ RMT (A), $120 \%$ RMT (B) and $150 \%$ RMT (C).

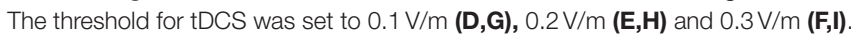

From the description of the direction of the E-field induced in TMS and tDCS, it is expected that the mean angle between the E-field induced by these two techniques is high. That was indeed the case in all the cortical regions considered (see Table 1, last column), especially the $\mathrm{HK}, \mathrm{PMv}$ and the circular regions located under the cathode (C, in Table 1). In the SMA and PMd, the mean angles were smaller.

\section{Influence of Tissue Anisotropy}

All the results presented before were obtained in the model with anisotropic conductivities in the GM and WM tissues. Modeling the tissues as isotropic, however, had little effect on the E-field distribution in both TMS and tDCS (regardless of electrode size). To quantify this we calculated the differences between the variables identified in Table $\mathbf{1}$ in the anisotropic models and the isotropic ones. As before, we performed this calculation for all the areas identified. The results, presented as a percentage of the values obtained in the anisotropic model, show that the maximum difference was of only $+3.9 \%$ (azimuthal angle in the SMA region for the model with the $35 \mathrm{~cm}^{2}$ electrode over $\mathrm{M} 1$ ) and the minimum difference was of $-0.2 \%$ (E-field's magnitude in the PMv region, for the tDCS model with the largest electrode over M1). The spatial distribution of the relative difference of the E-field's norm is shown in Figure 4 for both the TMS and tDCS models. The results indicate that effects of anisotropy, albeit always small, are larger in the tDCS models than in the TMS one.

\section{DISCUSSION}

\section{Differences in E-field Distribution in TMS and tDCS}

This study provides important insights into the E-field distribution in TMS and tDCS and how it affects neurons in the cortex. The most prominent feature of the E-field induced in TMS was the presence of two maxima at the top of the pre- and post-central gyri. Moving the coil anteriorly proved inefficient at decreasing the maximum over the post-central gyrus significantly. These results are consistent with what was found in other studies reporting that when the primary E-field (which depends only on coil orientation and geometry) is perpendicular to the local orientation of the gyrus, the total E-field is strongest at the gyrus (Thielscher et al., 2011). They also indicate that projections from the somato-sensory cortex to the primary motor cortex may be stimulated in response to the TMS pulse. The 


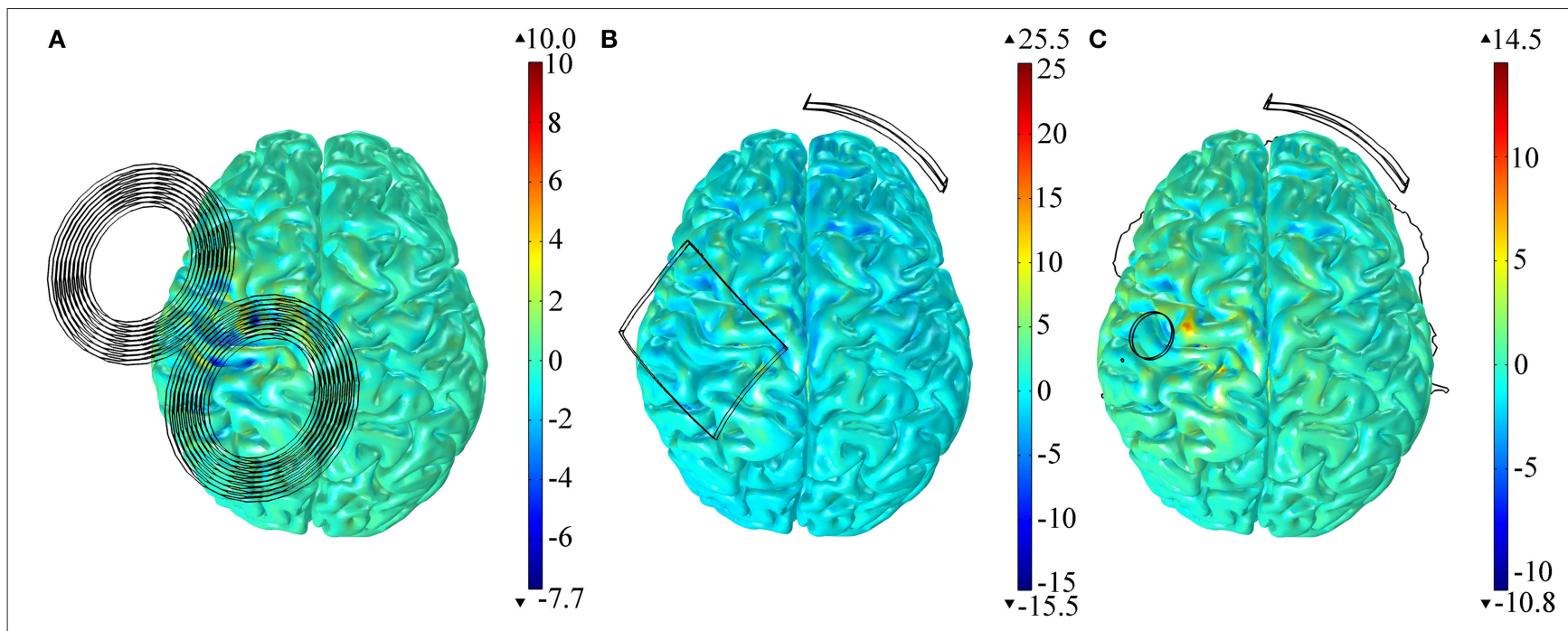

FIGURE 4 | Relative difference between the E-field magnitude distribution in the cortex in the anisotropic and the isotropic models for TMS (A), tDCS with the larger $35 \mathbf{c m}^{2}$ anode (B) and tDCS with the $\pi \mathbf{~ c m}^{2}$ anode (C). All results are presented as a percentage of the maximum E-field induced in the cortex in the anisotropic model.

latter have been suggested to play an important role in the results of TMS of the primary motor cortex (Esser et al., 2005; Salvador et al., 2011). Another important characteristic of the induced field is that it does not extend significantly along the depth of the anterior wall of the central sulcus, even with increasing stimulator output. Also, the induced field is mostly parallel to the cortical surface at the top of the gyri, and perpendicular to it in the sulci. Since only neuronal processes parallel to the field are affected by it (Roth, 1994), it is unlikely that the TMS-induced field directly stimulates pyramidal neurons at the top of the gyri, given that these are mostly oriented perpendicularly to the cortical surface (Kammer et al., 2007). In principle, stimulation could take place in the walls of the sulci, since there the field is aligned properly with pyramidal cells. However, the magnitude of the induced E-field on the sulcal walls decreases rapidly with depth so stimulation may occur only at stimulator outputs higher than RMT. The E-field at the top of the gyri will, however, have a strong effect on the cortical interneurons aligned mostly tangentially to the cortical surface and on collaterals of pyramidal cells with the same orientation (Nagarajan et al., 1993; Salvador et al., 2011). These structures, when aligned tangentially to the induced E-field, can be strongly polarized at their terminations.

The E-field induced in tDCS has very distinct characteristics to the one induced in TMS. One of these differences is its magnitude: the E-field's magnitude in $\mathrm{tDCS}$ is two to three orders of magnitude smaller than that of the field induced in TMS. Also, the location of the maxima of the E-field's magnitude is very different between the two techniques. Whereas in TMS the maxima are located at the top of the two gyri closest to the coil center, in tDCS with the $35 \mathrm{~cm}^{2}$ anode there is only one local maximum at the top of the precentral gyrus, together with global maxima located at the crowns of the gyri closest to the anterior edges of the anode and at the bottom of the sulci under the electrodes. This suggests that this
tDCS configuration may target not only motor but also pre-motor areas, as illustrated by the relatively high E-field values in these regions. The field induced in TMS, on the other hand, is much more localized in areas closer to the primary motor cortex. In the PMd there is also a strong maximum of the E-field induced in TMS, but its average magnitude is only $63 \%$ of the average over the HK region. In the tDCS model with the $\pi \mathrm{cm}^{2}$ electrode, the global maximum shifts to the crown of the pre-central gyrus, due to the fact that the edge of the electrode is located above it. For this electrode configuration, in the pre-motor areas, the average E-field magnitude values are always smaller than those obtained at the HK.

Another important difference between the two field distributions is related to the orientation of the E-field. In the motor cortex, the orientation of the E-field induced by these two techniques is remarkably different, with the E-field induced in tDCS (regardless of anode size and shape) having a component predominantly perpendicular to the cortical surface at the top of the gyri. This is shown by the mean values of the polar angle in the HK, which are higher in tDCS than in TMS. This indicates that tDCS probably targets preferentially neural processes perpendicular to the cortical surface at the top of the pre-central gyrus, such as pyramidal neurons. Since the latter have been described as the most likely target in tDCS (Radman et al., 2009), these results indicate that this electrode configuration is indeed capable of stimulating those cells in the HK. Contrary to TMS, in tDCS the E-field at the top of the post-central gyrus is always relatively low. However, this region may be targeted with the smaller anode if the threshold value for tDCS efficacy is set to a sufficiently low value (about $0.3 \mathrm{~V} / \mathrm{m}$ in this study). In the pre-motor areas, the orientation of the E-field induced by both techniques tends to be slightly more similar (especially in the SMA) but big differences can still be found. This is particularly visible in the much bigger polar angles obtained in the PMd and PMv. In the PMd, the large mean polar angle indicates 
that the E-field might target the same type of neural processes mentioned before in the case of the HK, i.e., structures located perpendicularly to the surface of the gyri. The interpretation is harder for the PMv region, since this region is located laterally in the head. For this region it is the mean azimuthal angle that better predicts whether the field in perpendicular or not to the cortical surface at the top of the gyri. The mean azimuthal angle at the PMv in the model with the $35 \mathrm{~cm}^{2}$ electrode is $52^{\circ}$ which indicates a strong left-right direction and, therefore, a strong inward component of the field perpendicular to the cortical surface at the top of the gyri. Interestingly, in the model with the $\pi \mathrm{cm}^{2}$ electrode the mean azimuthal angle is $126^{\circ}$ which indicates a perpendicular component but pointing outwards from the cortical surface at the top of the gyri. These results indicate that the E-field induced in tDCS is capable of affecting pyramidal cells in these pre-motor regions as well. In the SMA the E-field tends to be more parallel to the one induced in TMS which may indicate that in this region it is harder for tDCS to polarize the pyramidal cells perpendicular to the top of the gyral surface. Since several projections exist between pre-motor areas and the primary motor cortex (Dum and Strick, 1991), these results suggest that the PMd and PMv may be involved in the tDCS induced after-effects in motor cortex excitability.

\section{Effects of Tissue Conductivities and other Model Limitations}

The bulk of the results presented in this work were obtained from anisotropic models of tissue conductivity. Our analysis, however, showed no substantial differences in E-field distribution between isotropic and anisotropic tissue conductivity models. This may stem from the fact that this analysis is based on E-field values in the GM and that the GM is mostly isotropic. This insensitivity to tissue anisotropy is in line with what was reported in a previous study (Opitz et al., 2011). In this work all the data analysis was presented in the GM because all neuronal processes affected by the E-field are located there. The only exceptions are the bends of pyramidal cells as they enter into the WM, which have been shown to be a possible excitation site in TMS of the motor cortex (Amassian et al., 1992; Maccabee et al., 1998). However, it is expected that the polarization at the bends depends strongly on the E-field magnitude along the direction of the neuron in the GM (Roth, 1994). Since the WM has a much higher fractional anisotropy it is expected that the influence of anisotropy in E-field values there is higher.

Other studies point out the substantial effect of the conductivity of the skull in the E-field distribution during tDCS (Metwally et al., 2012; Opitz et al., 2015). In TMS it is expected that the influence of skull conductivity will have a small effect on the overall distribution of the E-field (Roth et al., 1991). Nonetheless, future tDCS work should focus on determining the effects of assigning different conductivity values to this tissue.

The way the stimulation electrodes were modeled in this work assumes that they are homogeneous conductive materials with a conductivity of $2 \mathrm{~S} / \mathrm{m}$. The conductivity of the material of the electrodes has been shown to have a negligible effect on the E-field distribution in the brain within the reasonable conductivity range of $0.2-20 \mathrm{~S} / \mathrm{m}$ (Opitz et al., 2015). The fact that the contact between the electrode and the skin is modeled as perfect is more likely to affect the prediction of the induced E-field, particularly in the scalp. The particular way in which the coil was modeled in the TMS study has also been shown to not be accurate for regions very close to the coil wires (Salinas et al., 2007). We expect, however, that these effects are not very pronounced for the E-field distribution in the brain.

Another important limitation of this work is that only one head model was created. The question of inter-subject variability in this experimental protocol has been demonstrated lately in some works (López-Alonso et al., 2014; Wiethoff et al., 2014). This shows the importance of considering different head models since anatomical changes, like layer thickness and gyri variability, can have a strong effect on the E-field distribution (Laakso et al., 2015; Opitz et al., 2015). This is clearly shown by the differences between overall E-field distribution between this head model and the one presented in our previous work (Miranda et al., 2013). In that work, the E-field distribution presented global maxima at the bottom of the sulci beneath the electrodes. In the present work, only local maxima are found there, and the E-field global maxima are found at the top of the gyri. This is more strongly seen in the model with the smaller $\pi \mathrm{cm}^{2}$ electrode over the HK. This is most likely related to the thickness of the skull and CSF being smaller in this model as compared to the one in the last study. Regarding the effects of the variability of the cortical surface geometry, it is expected that it has a stronger effect in the TMS E-field calculation, since charge accumulation at the GM-CSF interface seems to explain the patterns of maxima observable in the E-field induced during this type of stimulation (Thielscher et al., 2011).

Future work should focus on the determination of how the E-field distribution affects the membrane potential of multicompartmental neuron models. Some studies do exist featuring such models, but often they assume either a simplified neuronal geometry (Salvador et al., 2011) or E-fields applied along the neuron that were not obtained from realistic volume conductor models (Kamitani et al., 2001; Wu et al., 2013). Models including both a realistic representation of the neuronal geometry, and a calculation of the E-field in a realistic volume conductor model are, to the best of our knowledge, inexistent for $\mathrm{tDCS} / \mathrm{TMS}$. Combining the latter with models of large scale assemblies of neurons (Esser et al., 2005; Merlet et al., 2013; Molaee-Ardekani et al., 2013) should lead to further insights on the mechanisms of TMS and tDCS.

\section{FUNDING}

IBEB is funded by the Portuguese Foundation for Science and Technology (FCT), under project UID/BIO/00645/2013. CW is supported by Novocure.

\section{SUPPLEMENTARY MATERIAL}

The Supplementary Material for this article can be found online at: http://journal.frontiersin.org/article/10.3389/fncel. 2015.00405 


\section{REFERENCES}

Amassian, V. E., Eberle, L., Maccabee, P. J., and Cracco, R. Q. (1992). Modelling magnetic coil excitation of human cerebral cortex with a peripheral nerve immersed in a brain-shaped volume conductor: the significance of fiber bending in excitation. Electroencephalogr. Clin. Neurophysiol. 85, 291-301. doi: 10.1016/0168-5597(92)90105-K

Chen, M., and Mogul, D. J. (2009). A structurally detailed finite element human head model for simulation of transcranial magnetic stimulation. J. Neurosci. Methods 179, 111-120. doi: 10.1016/j.jneumeth.2009. 01.010

Comsol. (2013a). Comsol API for use with Java - Reference manual. Comsol.

Comsol. (2013b). Comsol multiphysics - Reference manual. Stockholm: Comsol, Inc.

Datta, A., Bansal, V., Diaz, J., Patel, J., Reato, D., and Bikson, M. (2009). Gyriprecise head model of transcranial direct current stimulation: improved spatial focality using a ring electrode versus conventional rectangular pad. Brain Stimul. 2, 201-207. doi: 10.1016/j.brs.2009.03.005

Dum, R. P., and Strick, P. L. (1991). The origin of corticospinal projections from the premotor areas in the frontal-lobe. J. Neurosci. 11, 667-689.

Esser, S. K., Hill, S. L., and Tononi, G. (2005). Modeling the effects of transcranial magnetic stimulation on cortical circuits. J. Neurophysiol. 94, 622-639. doi: 10.1152/jn.01230.2004

Kamitani, Y., Bhalodia, V. M., Kubota, Y., and Shimojo, S. (2001). A model of magnetic stimulation of neocortical neurons. Neurocomputing 38, 697-703. doi: 10.1016/S0925-2312(01)00447-7

Kammer, T., Beck, S., Thielscher, A., Laubis-Herrmann, U., and Topka, H. (2001). Motor thresholds in humans: a transcranial magnetic stimulation study comparing different pulse waveforms, current directions and stimulator types. Clin. Neurophysiol. 112, 250-258. doi: 10.1016/S1388-2457(00)00513-7

Kammer, T., Vorwerg, M., and Herrnberger, B. (2007). Anisotropy in the visual cortex investigated by neuronavigated transcranial magnetic stimulation. NeuroImage 36, 313-321. doi: 10.1016/j.neuroimage.2007.03.001

Laakso, I., Tanaka, S., Koyama, S., De Santis, V., and Hirata, A. (2015). Inter-subject variability in electric fields of motor cortical tDCS. Brain Stimul. 8, 906-913. doi: 10.1016/j.brs.2015.05.002

Liebetanz, D., Nitsche, M. A., Tergau, F., and Paulus, W. (2002). Pharmacological approach to the mechanisms of transcranial DC-stimulation-induced aftereffects of human motor cortex excitability. Brain 125, 2238-2247. doi: 10.1093/brain/awf238

López-Alonso, V., Cheeran, B., Río-Rodríguez, D., and Fernández-del-Olmo, M. (2014). Inter-individual variability in response to non-invasive brain stimulation paradigms. Brain Stimul. 7, 372-380. doi: 10.1016/j.brs.2014. 02.004

Maccabee, P. J., Nagarajan, S. S., Amassian, V. E., Durand, D. M., Szabo, A. Z., Ahad, A. B., et al. (1998). Influence of pulse sequence, polarity and amplitude on magnetic stimulation of human and porcine peripheral nerve. J. Physiol. 513, 571-585. doi: 10.1111/j.1469-7793.1998. $571 \mathrm{bb} . x$

Mayka, M. A., Corcos, D. M., Leurgans, S. E., and Vaillancourt, D. E. (2006). Three-dimensional locations and boundaries of motor and premotor cortices as defined by functional brain imaging: a metaanalysis. NeuroImage 31, 1453-1474. doi: 10.1016/j.neuroimage.2006. 02.004

Merlet, I., Birot, G., Salvador, R., Molaee-Ardekani, B., Mekonnen, A., Soria-Frisch, A., et al. (2013). From oscillatory transcranial current stimulation to scalp EEG changes: a biophysical and physiological modeling study. PLoS ONE 8:e57330. doi: 10.1371/journal.pone.0057330

Metwally, M. K., Cho, Y. S., Park, H. J., and Kim, T. S. (2012). Investigation of the electric field components of tDCS via anisotropically conductive gyrispecific finite element head models. Conf. Proc. IEEE Eng. Med. Biol. Soc. 2012, 5514-5517. doi: 10.1109/EMBC.2012.6347243

Miranda, P. C., Mekonnen, A., Salvador, R., and Ruffini, G. (2013). The electric field in the cortex during transcranial current stimulation. NeuroImage 70, 48-58. doi: 10.1016/j.neuroimage.2012.12.034

Molaee-Ardekani, B., Márquez-Ruiz, J., Merlet, I., Leal-Campanario, R., Gruart, A., Sánchez-Campusano, R., et al. (2013). Effects of transcranial Direct Current Stimulation (tDCS) on cortical activity: a computational modeling study. Brain Stimul. 6, 15. doi: 10.1016/j.brs.2011. 12.006

Nagarajan, S. S., Durand, D. M., and Warman, E. N. (1993). Effects of induced electric-fields on finite neuronal structures: a simulation study. IEEE Trans. Biomed. Eng. 40, 1175-1188. doi: 10.1109/10. 245636

Nitsche, M. A., Cohen, L. G., Wassermann, E. M., Priori, A., Lang, N., Antal, A., et al. (2008). Transcranial direct current stimulation: state of the art 2008. Brain Stimul. 1, 206-223. doi: 10.1016/j.brs.2008. 06.004

Nitsche, M. A., Fricke, K., Henschke, U., Schlitterlau, A., Liebetanz, D., Lang, N., et al. (2003). Pharmacological modulation of cortical excitability shifts induced by transcranial direct current stimulation in humans. J. Physiol. 553, 293-301. doi: 10.1113/jphysiol.2003.049916

Nitsche, M. A., and Paulus, W. (2000). Excitability changes induced in the human motor cortex by weak transcranial direct current stimulation. J. Physiol. 527, 633-639. doi: 10.1111/j.1469-7793.2000.t01-100633.x

Nitsche, M. A., and Paulus, W. (2001). Sustained excitability elevations induced by transcranial DC motor cortex stimulation in humans. Neurology 57, 1899-1901. doi: 10.1212/WNL.57.10.1899

Opitz, A., Legon, W., Rowlands, A., Bickel, W. K., Paulus, W., and Tyler, W. J. (2013). Physiological observations validate finite element models for estimating subject-specific electric field distributions induced by transcranial magnetic stimulation of the human motor cortex. NeuroImage 81, 253-264. doi: 10.1016/j.neuroimage.2013.04.067

Opitz, A., Paulus, W., Will, S., Antunes, A., and Thielscher, A. (2015). Determinants of the electric field during transcranial direct current stimulation. NeuroImage 109, 140-150. doi: 10.1016/j.neuroimage.2015. 01.033

Opitz, A., Windhoff, M., Heidemann, R. M., Turner, R., and Thielscher, A. (2011). How the brain tissue shapes the electric field induced by transcranial magnetic stimulation. NeuroImage 58, 849-859. doi: 10.1016/j.neuroimage.2011. 06.069

Plonsey, R., and Heppner, D. B. (1967). Considerations of quasi-stationarity in electrophysiological systems. Bull. Math. Biophys. 29, 657-664. doi: 10.1007/BF02476917

Radman, T., Ramos, R. L., Brumberg, J. C., and Bikson, M. (2009). Role of cortical cell type and morphology in subthreshold and suprathreshold uniform electric field stimulation in vitro. Brain Stimul. 2, 215-228. doi: 10.1016/j.brs.2009.03.007

Roth, B. J. (1994). Mechanisms for electrical-stimulation of excitable tissue. Crit. Rev. Biomed. Eng. 22, 253-305.

Roth, B. J., Cohen, L. G., and Hallett, M. (1991). The electric field induced during magnetic stimulation. Electroencephalogr. Clin. Neurophysiol. 43, 268-278.

Salinas, F. S., Lancaster, J. L., and Fox, P. T. (2007). Detailed 3D models of the induced electric field of transcranial magnetic stimulation coils. Phys. Med. Biol. 52, 2879-2892. doi: 10.1088/0031-9155/52/ $10 / 016$

Salvador, R., Silva, S., Basser, P. J., and Miranda, P. C. (2011). Determining which mechanisms lead to activation in the motor cortex: a modeling study of transcranial magnetic stimulation using realistic stimulus waveforms and sulcal geometry. Clin. Neurophysiol. 122, 748-758. doi: 10.1016/j.clinph.2010. 09.022

Thielscher, A., and Kammer, T. (2002). Linking physics with physiology in TMS: a sphere field model to determine the cortical stimulation site in TMS. NeuroImage 17, 1117-1130. doi: 10.1006/nimg.2002.1282

Thielscher, A., Opitz, A., and Windhoff, M. (2011). Impact of the gyral geometry on the electric field induced by transcranial magnetic stimulation. NeuroImage 54, 234-243. doi: 10.1016/j.neuroimage.2010. 07.061

Tofts, P. S. (1990). The distribution of induced currents in magnetic stimulation of the nervous-system. Phys. Med. Biol. 35, 1119-1128. doi: 10.1088/00319155/35/8/008

Wiethoff, S., Hamada, M., and Rothwell, J. C. (2014). Variability in response to transcranial direct current stimulation of the 
motor cortex. Brain Stimul. 7, 468-475. doi: 10.1016/j.brs.2014. 02.003

Wu, T. C., Jie, F., Seng, L. K., Li, X. P., and Wilder-Smith, E. P. V. (2013). "Modeling of membrane potential dynamics induced by electromagnetic stimulation," in 2013 Annual International Conference of the IEEE Engineering on Neural Engineering (San Diego), 243-246.

Yousry, T. A., Schmid, U. D., Alkadhi, H., Schmidt, D., Peraud, A., Buettner, A., et al. (1997). Localization of the motor hand area to a knob on the precentral gyrus. A new landmark. Brain 120, 141-157. doi: 10.1093/brain/ 120.1.141
Conflict of Interest Statement: The authors declare that the research was conducted in the absence of any commercial or financial relationships that could be construed as a potential conflict of interest.

Copyright (c) 2015 Salvador, Wenger and Miranda. This is an open-access article distributed under the terms of the Creative Commons Attribution License (CC BY). The use, distribution or reproduction in other forums is permitted, provided the original author(s) or licensor are credited and that the original publication in this journal is cited, in accordance with accepted academic practice. No use, distribution or reproduction is permitted which does not comply with these terms. 